\title{
On the generalized Wiener polarity index for some classes of graphs
}

\author{
Halina Bielak \\ Maria Curie - Skłodowska University \\ Pl. Marii Curie - Skłodowskiej 5 \\ 20-031 Lublin, Poland \\ Email: hbiel@hektor.umcs.lublin.pl
}

\author{
Kinga Dąbrowska, Katarzyna Wolska \\ Maria Curie - Skłodowska University \\ Pl. Marii Curie - Skłodowskiej 5 \\ 20-031 Lublin, Poland \\ Email: kinga.wiktoria.dabrowska@gmail.com, \\ katarzyna.anna.wolska@gmail.com
}

\begin{abstract}
The generalized Wiener polarity index $W_{k}(G)$ of a graph $G=(V, E)$ is defined as a number of unordered pairs $\{u, v\}$ of $G$ such that the shortest distance between $u$ and $v$ is equal to $k$ :

$$
W_{k}(G)=|\{\{u, v\}, d(u, v)=k, u, v \in V(G)\}|
$$

In this paper we give some results for 2-trees in case of mentioned index. We present an infinite family of 2 -trees with maximum value of generalized Wiener polarity index.
\end{abstract}

\section{INTRODUCTION}

$\mathbf{L}$ ET $G=(V(G), E(G))$ be a connected, simple graph with $V(G)$ the vertex set and $E(G)$ the edge set. Let $n$ be the number of vertices and $m$ the number of edges. By $d(u, v)$ we denote the distance between two vertices $u$ and $v$ in the graph $G$. What we call a diameter $\operatorname{diam}(G)$ is the longest distance between two vertices of $G$. The degree of the vertex $u$ in the graph $G$ is denoted by $\operatorname{deg}(u)$. Other definitions, not mentioned here can be found in [1].

The Wiener polarity index of a graph $G=(V(G), E(G))$ is defined as

$$
W P(G)=|\{\{u, v\}: d(u, v)=3 ; u, v \in V(G)\}|
$$

which is a number of unordered pairs of vertices $\{u, v\}$ of $G$ such that $d(u, v)=3$. Authors of $[4,5,7,13]$ studied this index for trees with different parameters such that number of pendant vertices, diameter or maximum degree. Additionally, in [12] there are described algorithms for counting $W_{k}(T)$ for trees.

The generalized Wiener polarity index of a graph $G=$ $(V(G), E(G))$ is defined as

$$
W_{k}(G)=|\{\{u, v\}, d(u, v)=k, u, v \in V(G)\}|
$$

which is a number of unordered pairs of vertices $\{u, v\}$ of $G$ such that the distance between $u$ and $v$ is equal to $k$.

Let us now remind the definition of the Wiener index $W(G)$

$$
W(G)=\sum_{\{u, v\} \subseteq V(G)} d(u, v)=\frac{1}{2} \sum_{v \in V(G)} D(v),
$$

where $D(v)=\sum_{u \in V(G)} d(u, v)$ is the sum of all distances from the vertex $v$. As we can see $W(G)$ is defined as the sum of the distances between all pairs of vertices in the graph $G$. Note that: $W(G)=\sum_{k=1}^{\operatorname{diam}(G)} k W_{k}(G)$. The Hosoya polynomial (Wiener polynomial) of $G$ in $x$ is defined as follows

$$
W(G, x)=\sum_{u, v \in V(G)} x^{d(u, v)}=\sum_{k=1}^{\operatorname{diam}(G)} W_{k}(G) \cdot x^{k}
$$

More information about Hosoya polynomial the reader can find in [9].

The applications of mentioned indices are described in the papers [2, 3] and also in [9, 10]. Probably the best known topological index is the Wiener index and this is the one described by many authors, for example $[2,8]$.

\section{Generalized WIENER POLARITY INDEX}

In case of generalized Wiener polarity index for trees there are some known results presented in [12]. Let $T$ be a tree. If $k=1$ then $W_{1}(T)=m$, where $m$ is the number of edges. If $k=2$ then

$$
\begin{aligned}
W_{2}(T) & =\sum_{v \in V(T)}\left(\begin{array}{c}
\operatorname{deg}(v) \\
2
\end{array}\right)=\frac{\sum_{v \in V(T)} \operatorname{deg}^{2}(v)}{2}-m \\
& =\frac{M_{1}(G)}{2}-m
\end{aligned}
$$

where $M_{1}(G)$ is the first Zagreb index of a graph. For detailed information on Zagreb indices the reader is referred to [11].

If $k=3$ we have

$$
\begin{aligned}
W_{3}(T) & =\sum_{u v \in E(T)}(\operatorname{deg}(v)-1)(\operatorname{deg}(u)-1) \\
& =\sum_{u v \in E(T)} \operatorname{deg}(u) \operatorname{deg}(v)-\sum_{v \in V(T)} \operatorname{deg}^{2}(v)+m \\
& =M_{2}(T)-M_{1}(T)+m
\end{aligned}
$$

where $M_{2}(T)$ is the second Zagreb index of a graph.

Let us now assume that $k \geq 3$. In a situation when diameter of $T$ is less than $k$ we have $W_{k}(T)=0$ and that is why the minimum value of $W_{k}(T)$ is equal to zero. This is 
achieved for all trees for which $\operatorname{diam}(T)<k$. Actually, this is simple fact for each graph.

Now we will study the generalized Wiener polarity index for 2-trees. Let us define a 2-tree first. The smallest 2-tree is a complete graph $K_{3}$ of order $n=3$. A 2-tree of order $n$ is obtained from a 2 -tree $G$ of order $n-1$ by attaching a new vertex $v$ and two edges $\{v x, v y\}$ such that $\{x, y\} \in E(G)$. Concerning 2-trees with $\operatorname{diam}(G) \geq k$ is more difficult than for trees.

Let $G$ be a 2 -tree of order $n$ and size $m$. A pendant vertex in a 2 -tree is a vertex with degree equal to 2 . Now, for $k=1$ the value of $W_{1}(G)$ stays the same as for trees. For $k=2$ we have

$$
W_{2}(G)=\sum_{v \in V(G)}\left(\left(\begin{array}{c}
\operatorname{deg}(v) \\
2
\end{array}\right)-m\right)
$$

But let us move on to what will be considered now and this are the maximum values of $W_{k}(G)$ where $G$ is a 2-tree.

What we are going to do is to decompose all vertices $v$ in $G$ with $\operatorname{deg}(v)=2$ into some number of groups. Each group has the following property

$$
A_{i}=\left\{v \in V(G): \operatorname{deg}(v)=2 \wedge \exists_{e_{i}=\left\{u_{i}, w_{i}\right\}} ; v u_{i}, v w_{i} \in E(G)\right\}
$$

for $i=1,2, \ldots$

We have at least two such groups. Let us say that the distance between two arbitrary pendant vertices from different groups is not equal to $k$. Distances between vertices in each group are equal to 2 .

Let $p_{1}$ and $p_{2}$ be the numbers of vertices on distance $k$ from an arbitrary pendant vertex from $A_{1}$ and $A_{2}$, respectively. We can ssume that $p_{1} \geq p_{2}$ with no loss of generality. After removal of all pendant vertices from $A_{2}$ and addition to the group $A_{1}$ we get the transformed 2-tree $G^{\prime}$

$$
\begin{aligned}
& W_{k}\left(G^{\prime}\right)-W_{k}(G) \geq \\
& \quad=\left(\left|A_{1}\right| p_{1}+\left|A_{2}\right| p_{1}\right)-\left(\left|A_{1}\right| p_{1}+\left|A_{2}\right| p_{2}\right)= \\
& \quad=\left|A_{2}\right|\left(p_{1}-p_{2}\right) \geq 0
\end{aligned}
$$

Note this is true for two groups. If there are more of them inequality in (1) may not hold.

By repetition of this transformation we will get a new 2-tree with possibly greater generalized Wiener polarity index. The diameter of $G^{\prime}$ after each transformation is less or stays the same as the one for $G$. Each transformation gives us also one new pendant vertex. If we will choose the most distant groups of pendant vertices we will get a 2-tree with diameter equal to $k$. After that we can apply the transformation finitely many times until all pendant vertices are on distance $k$ and no other vertex of the final 2-tree has eccentricity equal to $k$. During this process the $W_{k}(G)$ may be changing by decreasing or increasing. Some example is presented in Fig.1.

Let us assume we have $p$ groups of pendant vertices with sizes: $a_{1}, a_{2}, \ldots, a_{p}$ and $a_{1}+a_{2}+\ldots+a_{p}=q$. We consider a 2 tree with $\operatorname{diam}(G)=k$. We have then $n-2(k-1) \geq q \geq 2$.
Assume that the distance between any two pendant vertices not from the same group is equal to $k$ and that is why

$$
W_{k}(G)=\frac{1}{2} \sum_{i=1}^{p} a_{i}\left(q-a_{i}\right)=\frac{1}{2}\left(q^{2}-\sum_{i=1}^{p} a_{i}^{2}\right)
$$

In the case when the distance between the group $A_{i}$ and $A_{j}$ for $i \neq j$ is less than $k$ the generalized Wiener polarity index is less than the one presented above. If $p=2$ we have $W_{k}(G)=$ $a_{1} a_{2}$. This value is maximum for $a_{1}+a_{2}=n-2(k-1)$, $a_{1}=\left\lfloor\frac{n-2(k-1)}{2}\right\rfloor$ and $a_{2}=\left\lceil\frac{n-2(k-1)}{2}\right\rceil$.

$$
\begin{aligned}
& W_{k}(G)=\left\lfloor\frac{n-2(k-1)}{2}\right\rfloor\left\lceil\frac{n-2(k-1)}{2}\right\rceil= \\
& =\left(\left\lfloor\frac{n}{2}\right\rfloor-(k-1)\right)\left(\left\lceil\frac{n}{2}\right\rceil-(k-1)\right)= \\
& =\left\lfloor\frac{n}{2}\right\rfloor\left\lceil\frac{n}{2}\right\rceil-(k-1)\left(\left\lfloor\frac{n}{2}\right\rfloor+\left\lceil\frac{n}{2}\right\rceil\right)+(k-1)^{2}
\end{aligned}
$$

so

$$
W_{k}(G)=\left\lfloor\frac{n}{2}\right\rfloor\left\lceil\frac{n}{2}\right\rceil-(k-1)(n-(k-1))
$$

Let $p>2$ and $k>2$. First we consider the even $k$. By $n \geq 2+p(k-2)+q$ and $2<p \leq q$ we have $p \leq \frac{n-2-q}{k-2}$, so

$$
p<\frac{n-2}{k-2} \text {. }
$$

We have the following

$$
\begin{gathered}
q=\sum_{i=1}^{p} a_{i}, \\
n=2+2 p\left(\frac{k}{2}-1\right)+\sum_{i=1}^{p} a_{i}=2+p(k-2)+\sum_{i=1}^{p} a_{i} .
\end{gathered}
$$

Hence

$$
n \geq 2+p(k-2)+q
$$

We apply Cauchy - Schwarz ineqality to the formula (2) with $q \leq n-2-p(k-2)$

$$
W_{k}(G)=\frac{1}{2}\left(q^{2}-\sum_{i=1}^{p} a_{i}^{2}\right) \leq \frac{1}{2}\left(q^{2}-\frac{q^{2}}{p}\right) \leq \frac{1}{2} f(p)
$$

where

$$
f(p)=(n-2-p(k-2))^{2}\left(1-\frac{1}{p}\right) .
$$

The extremal generalized Wiener polarity index $W_{k}(G)$ is obtained for the case when we have equality in (6). We are going to study this case. We will give some examples of extremal 2-trees and then we will state the final result in Theorem 1.

So for real variable $p$ we study

$$
\begin{aligned}
f(p)= & \left((n-2)^{2}+p^{2}(k-2)^{2}\right)\left(1-\frac{1}{p}\right) \\
& -2(k-2)(n-2)(p-1) .
\end{aligned}
$$



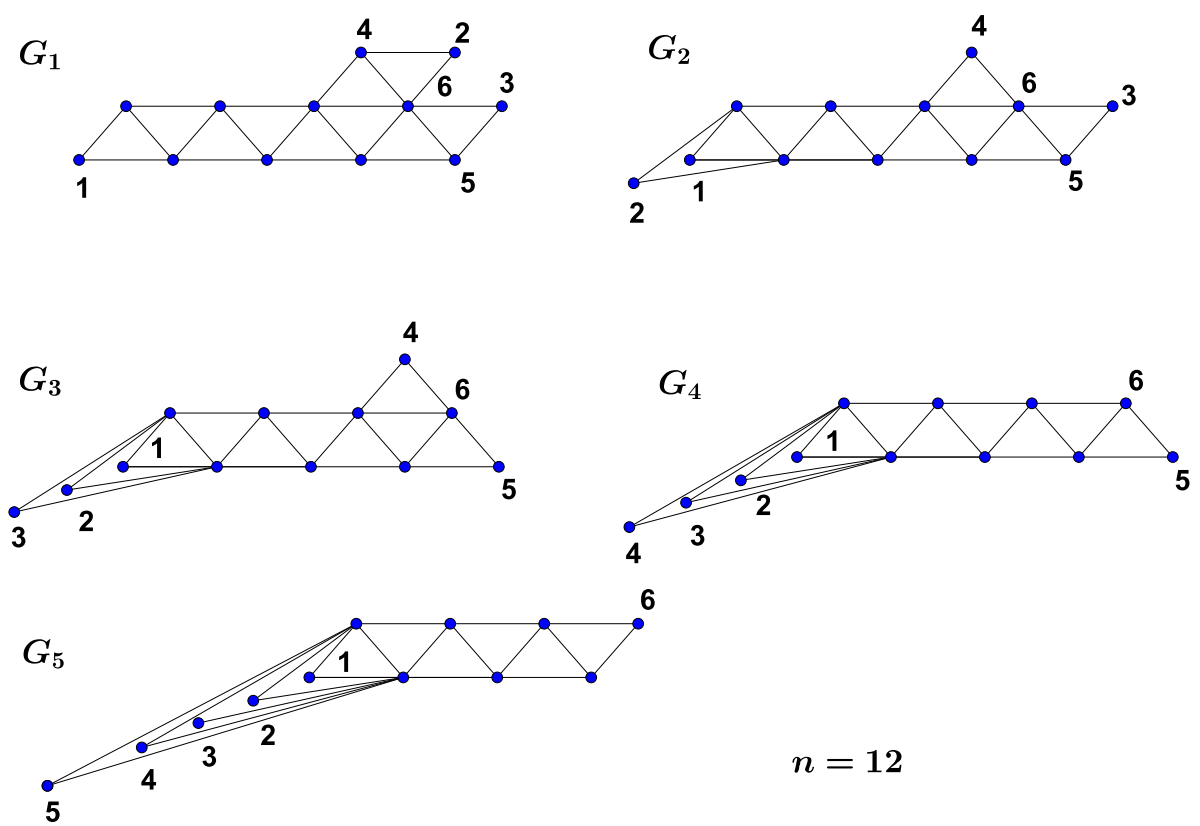

Fig. 1. A process of moving pendant vertices $G_{1} \rightarrow G_{2} \rightarrow G_{3} \rightarrow G_{4} \rightarrow G_{5} . W_{4}\left(G_{1}\right)=8, W_{4}\left(G_{2}\right)=9, W_{4}\left(G_{3}\right)=10, W_{4}\left(G_{4}\right)=9, W_{4}\left(G_{5}\right)=5$.

Let $h(p)=2(2-k)\left(1-\frac{1}{p}\right)+\frac{n-2-p(k-2)}{p^{2}}$. Then the first derivative equals

$$
f^{\prime}(p)=(n-2-p(k-2)) h(p)
$$

By (4) we have $f^{\prime}(p)=0$ if and only if $p=\hat{p}$, where

$$
\hat{p}=\frac{1}{4}+\frac{1}{4} \sqrt{\frac{8 n+k-18}{k-2}}
$$

Similarly $f^{\prime}(p)>0$ if and only if $h(p)>0$. This is equivalent to the inequality

$$
g(p)=2 p^{2}-p-\frac{n-2}{k-2}<0 .
$$

So $g(p)<0$ if and only if $p<\hat{p}$.

Let

$$
s=4 \hat{p}=1+\sqrt{\frac{8 n+k-18}{k-2}} .
$$

Then

$\frac{1}{\hat{p}}=\frac{\sqrt{(k-2)(8 n+k-18)}-(k-2)}{2 n-4}=\frac{(k-2)(s-2)}{2 n-4}$.

By (6) we can write

$$
f(\hat{p})=\left(n-2-\frac{1}{4}(k-2) s\right)^{2}\left(1-\frac{(k-2)(s-2)}{2 n-4}\right) .
$$

Then

$$
\begin{aligned}
f(\hat{p})= & \left((n-2)^{2}-\frac{(n-2)(k-2)}{2} s+\frac{(k-2)^{2}}{16} s^{2}\right) . \\
& \left(1-\frac{(k-2)(s-2)}{2 n-4}\right) .
\end{aligned}
$$

We are interested in the case with $\hat{p} \geq 3$. By (8) we get $n \geq 15 k-28$.

\section{Example 1:}

By the formula (9) for $k=6$ we get

$$
\begin{aligned}
& f(\hat{p})= \\
& \quad\left((n-2)^{2}-2(n-2)(1+\sqrt{2 n-3})+(1+\sqrt{2 n-3})^{2}\right) . \\
& \quad\left(1-\frac{2 \sqrt{2 n-3}-2}{n-2}\right) .
\end{aligned}
$$

Let us set

$$
n=2 t^{2}+2 \geq 15 k-28=62 .
$$

By (8) for even $t$ we have

$$
\lfloor\hat{p}\rfloor=\left\lfloor\frac{1}{4}+\frac{1}{4} \sqrt{4 t^{2}+1}\right\rfloor=\left\lfloor\frac{1}{4}+\frac{t}{2}\right\rfloor=\frac{t}{2} .
$$

Note that by (7)

$$
f(\lfloor\hat{p}\rfloor)=4 t(t-1)^{2}(t-2),
$$

and

$$
f(\lceil\hat{p}\rceil)=4 t\left(t^{2}-t-2\right)^{2} \frac{1}{t+2}>f(\lfloor\hat{p}\rfloor),
$$

for $t>2$.

We can note that by the formula (6) we get extremal 2-trees for the case

$$
W_{6}(G)=\frac{1}{2} f(\lfloor\hat{p}\rfloor) .
$$


Now we compare this value with $W_{k}(G)$ for $p>2$. By the formula (3) for $p=2$ we get

$$
W_{6}(G) \leq\left(t^{2}+1\right)^{2}-5\left(2 t^{2}+2\right)+25=t^{4}-8 t^{2}+16 .
$$

So we get the following inequality

$$
\frac{1}{2} f(\lfloor\hat{p}\rfloor)>t^{4}-8 t^{2}+16
$$

By the formula (12) we get

$$
2 t(t-2)(t-1)^{2}>t^{4}-8 t^{2}+16 .
$$

The inequality (13) is equivalent to the following one

$$
2 t(t-1)^{2}>(t-2)^{2}(t+2)
$$

Suppose now that $t=6$, then by (10) we have $n=2 \cdot 6^{2}+$ $2=74$ and by (9) we have $\lfloor\hat{p}\rfloor=3$. The inequality (14) holds in this case. So we obtained the maximum $W_{6}(G)=3 \cdot 20^{2}$ for the 2 -tree $G$ with parameters $n=74, k=6, p=3$ and $\left|A_{i}\right|=(n-14) / 3=20$.

An extremal 2-tree is presented below in Fig. 2.

\section{Example 2:}

By the formula (9) for $k=4$ we get

$$
\begin{aligned}
& f(\hat{p})= \\
& \quad\left((n-2)^{2}-(n-2)(1+\sqrt{4 n-7})+\frac{1}{4}(1+\sqrt{4 n-7})^{2}\right) . \\
& \quad\left(1-\frac{\sqrt{4 n-7}-1}{n-2}\right) .
\end{aligned}
$$

We have:

$$
n=t^{2}+2 \geq 15 k-28=15 \cdot 4-28=32 .
$$

By (8) for even $t$ we have

$$
\lfloor\hat{p}\rfloor=\left\lfloor\frac{1}{4}+\frac{1}{4} \sqrt{4 t^{2}+1}\right\rfloor=\left\lfloor\frac{1}{4}+\frac{t}{2}\right\rfloor=\frac{t}{2} .
$$

and then by (7)

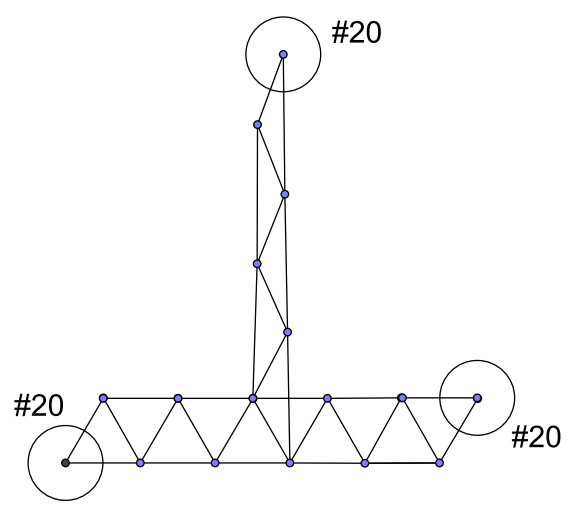

Fig. 2. An extremal graph of order $n=74$ with $k=6$ and three groups $\left|A_{i}\right|=20, i=1,2,3$.

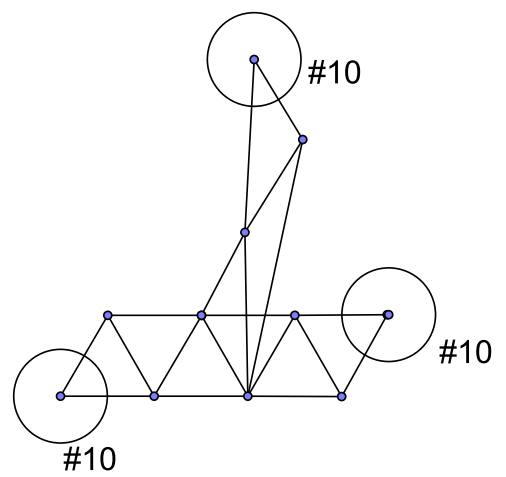

Fig. 3. An example of extremal graph for $k=4$.

$$
f(\lfloor\hat{p}\rfloor)=t(t-2)(t-1)^{2}
$$

and

$$
f(\lceil\hat{p}\rceil)=t\left(t^{2}-t-2\right)^{2} \frac{1}{t+2},
$$

We can note that by the formula (6) we get

$$
W_{4}(G)=\frac{1}{2} f(\lfloor\hat{p}\rfloor) .
$$

By the formula (3) for $p=2$ and $k=4$ we get $W_{4}(G)=$ $\frac{1}{4} t^{4}-2 t^{2}+4$.

Now we get the following inequality

$$
f(\lfloor\hat{p}\rfloor)=t(t-2)(t-1)^{2}>t\left(t^{2}-t-2\right)^{2} \frac{1}{t+2}=f(\lceil\hat{p}\rceil) .
$$

The above inequality is equivalent to the following one

$$
(t+2)(t-2)(t-1)^{2}>\left(t^{2}-t-2\right)^{2} \text {. }
$$

Suppose now that $t=6$, then by (15) we have $n=6^{2}+2=38>32$ and $\lfloor\hat{p}\rfloor=3$. So the inequality (17) holds in this case and we have the maximum $W_{4}(G)=3 \cdot 10^{2}$ for the 2-tree $G$ with parameters $n=38, k=4, \hat{p}=3$ and $\left|A_{i}\right|=(n-8) / 3=10, i=1,2,3$.

An extremal 2-tree is presented in Fig. 3.

In general case we have the following result.

Let $p_{-}=\lfloor\hat{p}\rfloor$ and $p_{+}=\lceil\hat{p}\rceil$ where $\hat{p}$ is defined in (8). We present a theorem for 2-trees of order $n$ equal to $g(k)$, where $g(k)$ is some function defined in the proof.

Theorem 1. Let $n$ and $k$ be integers. For each even integer $k \geq 4$ there exists a 2 -tree $G$ of order $n$ with extremal generalized Wiener polarity index $W_{k}(G)$ and with $p_{-} \geq 3$ or $p_{+} \geq 3$ groups of pendant vertices for $n=g(k)$ where $g(k)$ is some function in variable $k$. Then we have an infinite family of such 2-trees.

Proof: By (7) and (8) we have $p_{-} \leq \hat{p} \leq p_{+}$and $W_{k}(G)=$ $\frac{1}{2} \max \left\{f\left(p_{+}\right), f\left(p_{-}\right)\right\}$, where

$$
\begin{aligned}
& f\left(p_{-}\right)=\left((n-2)^{2}+p_{-}^{2}(k-2)^{2}\right)\left(1-\frac{1}{p_{-}}\right) \\
& -2(k-2)(n-2)\left(p_{-}-1\right) .
\end{aligned}
$$



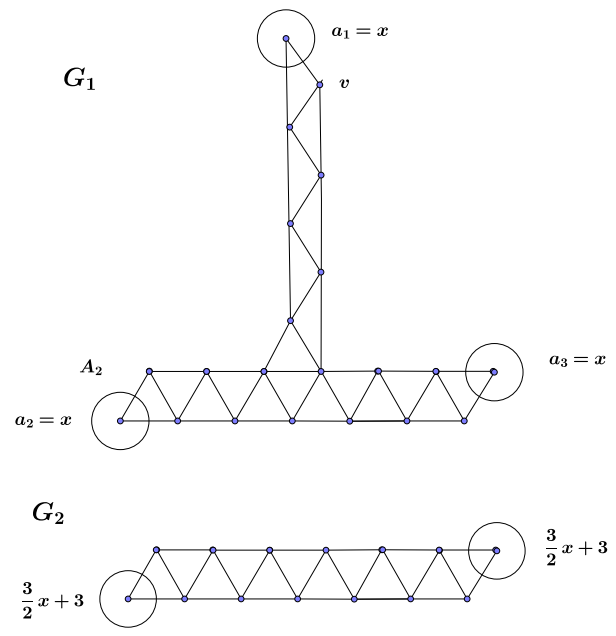

Fig. 4. Examples of 2-trees of the same order with diameter $k=7$, where $W_{7}\left(G_{1}\right)>W_{7}\left(G_{2}\right)$ for even $x \geq 12$.

We get the inequality

$$
\frac{1}{2} f\left(p_{-}\right)>\left\lfloor\frac{n}{2}\right\rfloor\left\lceil\frac{n}{2}\right\rceil-(k-1)(n-(k-1)) .
$$

Hence

$$
\begin{aligned}
& p_{-}^{3}(k-2)^{2}-p_{-}^{2}(k-2)(2 n+k-6) \\
& +p_{-}\left((n-2)^{2}-2\left\lfloor\frac{n}{2}\right\rfloor\left\lceil\frac{n}{2}\right\rceil+4 k n-6 n-2 k^{2}+6\right) \\
& -(n-2)^{2}>0 .
\end{aligned}
$$

Similarly we can compare

$$
\frac{1}{2} f\left(p_{+}\right)>\left\lfloor\frac{n}{2}\right\rfloor\left\lceil\frac{n}{2}\right\rceil-(k-1)(n-(k-1)) .
$$

This two above inequalities are equivalent to the following one:

$$
\begin{aligned}
& n^{2}-n(12((p-2) k-2 p)+52)+52-12 k^{2} \\
& +6 p\left((p-2)(k-2)^{2}+(k-2)(k+2)\right)>0
\end{aligned}
$$

where $p=p_{+}$or $p=p_{-}$.

By solving this inequality we can construct 2 -trees $G$ with $p_{-} \geq 3$ or $p_{+} \geq 3$ groups of pendant vertices with extremal generalized Wiener polarity index $W_{k}(G)$. It is enough to take $g(k)=2+p(k-2+a)$, where $a=\left|A_{i}\right|$ for each integer $a \geq(k-2) \max \{11,2(p-1)\}$ and $i=1, \ldots, p$ with $p=p_{-}$ or $p=p_{+}$. It follows by formula (8). This is the end of the proof.

In the theorem we are presenting the results for even $k$. Note that for odd $k$ the generalized Wiener polarity index $W_{k}(G)$ for 2 -trees of order $n$ with two groups of pendant vertices in general case is not greater than such index for 2 -trees of order $n$ with $p=3$ groups of pendant vertices.

An infinite number of such examples of 2-trees is presented in Fig. 4.

In this paper we proved Theorem 1 for 2-trees of order $n$ with an extremal $W_{k}(G)$ for given $n$ and $k$. In the future work we wish to find an efficient algorithm for counting $W_{k}(G)$ for the considered family of graphs.

\section{REFERENCES}

[1] Bondy, J. A., Murty, U. S. R., Graph Theory with Applications, Macmillan London and Elsevier, New York, 1976

[2] Chepoi, V., Klavžar, S.: The Wiener index and the Szeged index of benzenoid systems in linear time. J. Chem. Inf. Comput. Sci. 37, 752755 (1997); DOI: 10.1021/ci9700079

[3] Deng, H.: On the extremal Wiener polarity index of chemical trees.MATCH Commun. Math. Comput. Chem. 60, 305-314 (2011)

[4] Deng, H., Xiao, H., Tang, F.: The maximum Wiener polarity index of trees with k pendants. Appl. Math. Lett. 23, 710-715 (2010); DOI: 10.1016/j.aml.2010.02.013

[5] Deng, H., Xiao, H., Tang, F.: On the extremal Wiener polarity index of trees with a given diameter. MATCH Commun. Math. Comput. Chem. 63, 257-264 (2010) 123

[6] Deng, X., Zhang, J.: Equiseparability on terminal Wiener index. In: Goldberg, A.V., Zhou, Y. (eds.) Algorithmic Aspects in Information and Management, pp. 166-174. Springer, Berlin (2009); DOI: 10.1007/9783-642-02158-9 15

[7] Du,W., Li, X., Shi, Y.: Algorithms and extremal problem on Wiener polarity index.MATCH Commun. Math. Comput. Chem. 62, 235-244 (2009)

[8] Dobrynin, A.A., Entringer, R.C., Gutman, I.: Wiener index of trees: theory and applications. Acta Appl. Math. 66, 211-249 (2001); DOI: 10.1023/A:1010767517079

[9] Gutman, I., Zhang, Y., Dehmer, M., Ilić, A.: Altenburg,Wiener, and Hosoya polynomials. In: Gutman, I., Furtula, B. (eds.) Distance in Molecular Graphs-Theory, pp. 49-70. Univerity of Kragujevac, Kragujevac (2012)

[10] Hosoya, H.: Mathematical and chemical analysis of Wiener's polarity number. In: Rouvray, D.H., King, R.B. (eds.) Topology in ChemistryDiscrete Mathematics of Molecules. Horwood, Chichester (2002); DOI: 10.1533/9780857099617.38

[11] Ilić, A., Stevanović, D.: On Comparing Zagreb Indices. MATCH Commun. Math. Comput. Chem. 62, 681-687 (2009)

[12] Ilić, A., Ilić, M.: Generalizations of Wiener Polarity Index and Terminal Wiener Index, Graphs and Combinatorics 29, 1403-1416; 2013; DOI 10.1007/s00373-012-1215-6

[13] Liu, B., Hou, H., Huang, Y.: On the Wiener polarity index of trees with maximum degree or given number of leaves. Comp. Math. Appl. 60, 2053-2057 (2010); DOI:10.1016/j.camwa.2010.07.045 